\title{
Impact of future HERA data on the determination of proton parton distribution functions using the ZEUS NLO QCD fit
}

\author{
Claire Gwenlan†, Amanda Cooper-Sarkar $\dagger$ and Christopher Targett-Adams $\ddagger$ \\ $\dagger$ Department of Physics, Nuclear and Astrophysics Laboratory, Keble Road, Oxford. OX1 3RH. UK \\ $\ddagger$ Department of Physics and Astronomy, University College London, Gower Street, London. WC1E \\ 6BT. UK.
}

\begin{abstract}
The high precision and large kinematic coverage of the data from the HERA-I running period (1994-2000) have already allowed precise extractions of proton parton distribution functions. The HERA-II running program is now underway and is expected to provide a substantial increase in the luminosity collected at HERA. In this paper, a study is presented which investigates the potential impact of future data from HERA on the proton PDF uncertainties, within the currently planned running scenario. Next-to-leading order QCD predictions for inclusive jet cross sections at the LHC centre-of-mass energy are presented using the estimated PDFs. Finally, the effect of a possible future measurement of the longitudinal structure function, $F_{\mathrm{L}}$, on the gluon distribution is investigated.
\end{abstract}

\section{Introduction}

Since the advent of HERA, much progress has been made in determining the Parton Distribution Functions (PDFs) of the proton. A knowledge of the PDFs is vital in order to make predictions for both Standard Model and beyond the Standard Model processes at hadronic colliders. Furthermore, they must be known as precisely as possible in order to maximise the discovery potential for new physics at the LHC. HERA is now in its second stage of operation. With the measurements that can now be expected from HERA-II, our knowledge of PDFs should be further improved. In this paper, first studies of the potential impact on the PDF uncertainties of future measurements from HERA, are presented.

\section{HERA physics and kinematics}

Lepton-proton deep inelastic scattering (DIS) can proceed either via the neutral current (NC) interaction (through the exchange of a $\gamma^{*}$ or $\mathrm{Z}^{0}$ ), or via the charged current (CC) interaction (through the exchange of a $\mathrm{W}^{ \pm}$). The kinematics of lepton-proton DIS are described in terms of the Bjorken scaling variable, $x$, the negative invariant mass squared of the exchanged vector boson, $Q^{2}$, and the fraction of energy transferred from the lepton to the hadron system, $y$. The three quantities are related by $Q^{2}=s x y$, where $s$ is the centre-of-mass energy squared.

At leading order (LO) in the electroweak interaction, the double differential cross section for the NC DIS process is given in terms of proton structure functions,

$$
\frac{\mathrm{d}^{2} \sigma^{\mathrm{NC}}\left(e^{ \pm} p\right)}{\mathrm{d} x \mathrm{~d} Q^{2}}=\frac{2 \pi \alpha^{2}}{Q^{4} x}\left[Y_{+} \mathrm{F}_{2}\left(x, Q^{2}\right)-y^{2} \mathrm{~F}_{\mathrm{L}}\left(x, Q^{2}\right) \mp Y_{-} x \mathrm{~F}_{3}\left(x, Q^{2}\right)\right]
$$

where $Y_{ \pm}=1 \pm(1-y)^{2}$. The structure functions are directly related to the PDFs and their $Q^{2}$ dependence is predicted by perturbative $\mathrm{QCD}$. In particular, $\mathrm{F}_{2}$ and $x \mathrm{~F}_{3}$ depend directly on the quark distributions. For $Q^{2} \lesssim 1000 \mathrm{GeV}^{2}, \mathrm{~F}_{2}$ dominates the lepton-proton scattering cross section and for $x<10^{-2}, \mathrm{~F}_{2}$ itself is dominated by sea quarks while the $Q^{2}$ dependence is driven by gluon radiation. Therefore, HERA data provide vital information on the sea-quarks and gluon at low- $x$. At high $Q^{2} \gtrsim M_{\mathrm{Z}}^{2}$, the contribution 


\begin{tabular}{llcc}
\hline data sample & kinematic coverage & $\begin{array}{c}\text { HERA-I } \\
\mathcal{L}\left(\mathrm{pb}^{-1}\right)\end{array}$ & $\begin{array}{c}\text { HERA-II } \\
\mathcal{L}\left(\mathrm{pb}^{-1} \text { ) }\right. \\
\text { (assumed) }\end{array}$ \\
\hline 96-97 $\mathrm{NC} e^{+} p[7]$ & $2.7<Q^{2}<30000 \mathrm{GeV}^{2} ; 6.3 \cdot 10^{-5}<x<0.65$ & 30 & 30 \\
94-97 CC $e^{+} p[10]$ & $280<Q^{2}<17000 \mathrm{GeV}^{2} ; 6.3 \cdot 10^{-5}<x<0.65$ & 48 & 48 \\
98-99 NC $e^{-} p[8]$ & $200<Q^{2}<30000 \mathrm{GeV}^{2} ; 0.005<x<0.65$ & 16 & 350 \\
98-99 CC $e^{-} p[11]$ & $280<Q^{2}<17000 \mathrm{GeV}^{2} ; 0.015<x<0.42$ & 16 & 350 \\
99-00 NC $e^{+} p$ [9] & $200<Q^{2}<30000 \mathrm{GeV}^{2} ; 0.005<x<0.65$ & 63 & 350 \\
99-00 CC $e^{+} p[12]$ & $280<Q^{2}<17000 \mathrm{GeV}^{2} ; 0.008<x<0.42$ & 61 & 350 \\
96-97 inc. DIS jets [13] & $125<Q^{2}<30000 \mathrm{GeV}^{2} ; E_{\mathrm{T}}^{B r e i t}>8 \mathrm{GeV}$ & 37 & 500 \\
96-97 dijets in $\gamma p[14]$ & $Q^{2} \lesssim 1 \mathrm{GeV}^{2} ; E_{\mathrm{T}}^{\text {jet } 1,2}>14,11 \mathrm{GeV}$ & 37 & 500 \\
\hline optimised jets [17] & $Q^{2} \lesssim 1 \mathrm{GeV}^{2} ; E_{\mathrm{T}}^{\text {jet1,2 }}>20,15 \mathrm{GeV}$ & - & 500 \\
\hline
\end{tabular}

Table 1: The data-sets included in the ZEUS-JETS and HERA-II projected PDF fits. The first column lists the type of data and the second gives the kinematic coverage. The third column gives the integrated luminosities of the HERA-I measurements included in the ZEUS-JETS fit. The fourth column gives the luminosities assumed in the HERA-II projection. Note that the 96-97 NC and the 94-97 CC measurements have not had their luminosity scaled for the HERA-II projection.

from $x \mathrm{~F}_{3}$ becomes increasingly significant and gives information on the valence quark distributions. The longitudinal structure function, $\mathrm{F}_{\mathrm{L}}$, is directly sensitive to the gluon, but is only important at high- $y$.

At $\mathrm{LO}$, the $\mathrm{CC}$ cross sections are given by,

$$
\begin{aligned}
& \frac{\mathrm{d}^{2} \sigma^{\mathrm{CC}}\left(e^{+} p\right)}{\mathrm{d} x \mathrm{~d} Q^{2}}=\frac{G_{F}^{2} M_{W}^{4}}{2 \pi x\left(Q^{2}+M_{W}^{2}\right)^{2}} x\left[(\bar{u}+\bar{c})+(1-y)^{2}(d+s)\right] \\
& \frac{\mathrm{d}^{2} \sigma^{\mathrm{CC}}\left(e^{-} p\right)}{\mathrm{d} x \mathrm{~d} Q^{2}}=\frac{G_{F}^{2} M_{W}^{4}}{2 \pi x\left(Q^{2}+M_{W}^{2}\right)^{2}} x\left[(u+c)+(1-y)^{2}(\bar{d}+\bar{s})\right]
\end{aligned}
$$

so that a measurement of the $e^{+} p$ and $e^{-} p$ cross sections provides information on the $d$ - and $u$-valence quarks, respectively, thereby allowing the separation of flavour.

The QCD scaling violations in the inclusive cross section data, namely the QCD Compton $\left(\gamma^{*} q \rightarrow\right.$ $g q)$ and boson-gluon-fusion $\left(\gamma^{*} g \rightarrow q \bar{q}\right)$ processes, may also give rise to distinct jets in the final state. Jet cross sections therefore provide a direct constraint on the gluon through the boson-gluon-fusion process.

\section{PDF fits to HERA data}

PDFs are usually determined in global fits [1-3] made within the conventional DGLAP [4] formalism at next-to-leading order (NLO). Such fits use data from many different experiments, with the inclusive cross sections from DIS experiments providing the most important source of information. However, the high precision and wide kinematic coverage of the existing HERA data have already allowed precise extractions of the proton PDFs using only HERA data [5,6]. The high statistics HERA neutral current data determine the low- $x$ sea and gluon distributions while information on the valence quark distributions is provided by the higher- $Q^{2}$ neutral and charged current data. The gluon PDF contributes only indirectly to the inclusive DIS cross section. However, it makes a direct contribution to jet cross sections.

Recently, the ZEUS Collaboration have performed a combined NLO QCD fit to inclusive neutral and charged current DIS data [7-12] as well as high precision jet data in DIS [13] and $\gamma p$ scattering [14]. This is called the ZEUS-JETS PDF fit [5]. The use of only HERA data eliminates the uncertainties from heavy-target corrections and removes the need for isospin symmetry assumptions. It also avoids the difficulties that can sometimes arise from combining data-sets from several different experiments, thereby allowing a rigorous statistical treatment of the PDF uncertainties. Furthermore, PDF uncertainties from current global fits are, in general, limited by (irreducible) experimental systematics. In contrast, those 

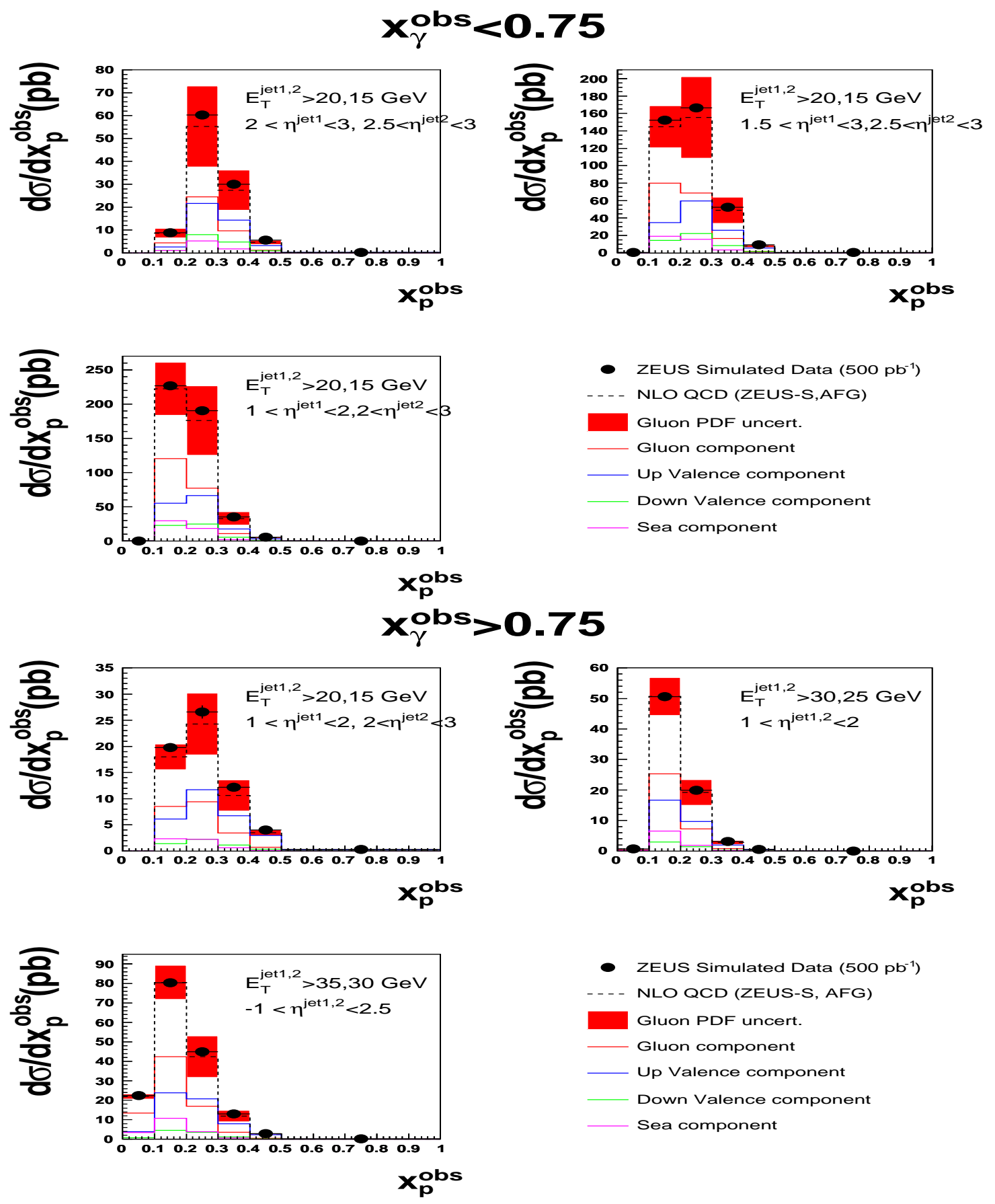

Fig. 1: The optimised jet cross sections included in the HERA-II projected fit. The solid points show the simulated data generated using the NLO QCD programme of Frixione-Ridolfi, using the CTEQ5M1 proton and the AFG photon PDFs. The error bars show the statistical uncertainties, which correspond to $500 \mathrm{pb}^{-1}$ of HERA data. Systematic uncertainties have been neglected. The dashed line shows the NLO QCD prediction using the ZEUS-S proton and AFG photon PDFs. The shaded band shows the contribution to the cross section uncertainty arising from the uncertainty in the gluon distribution in the proton. 

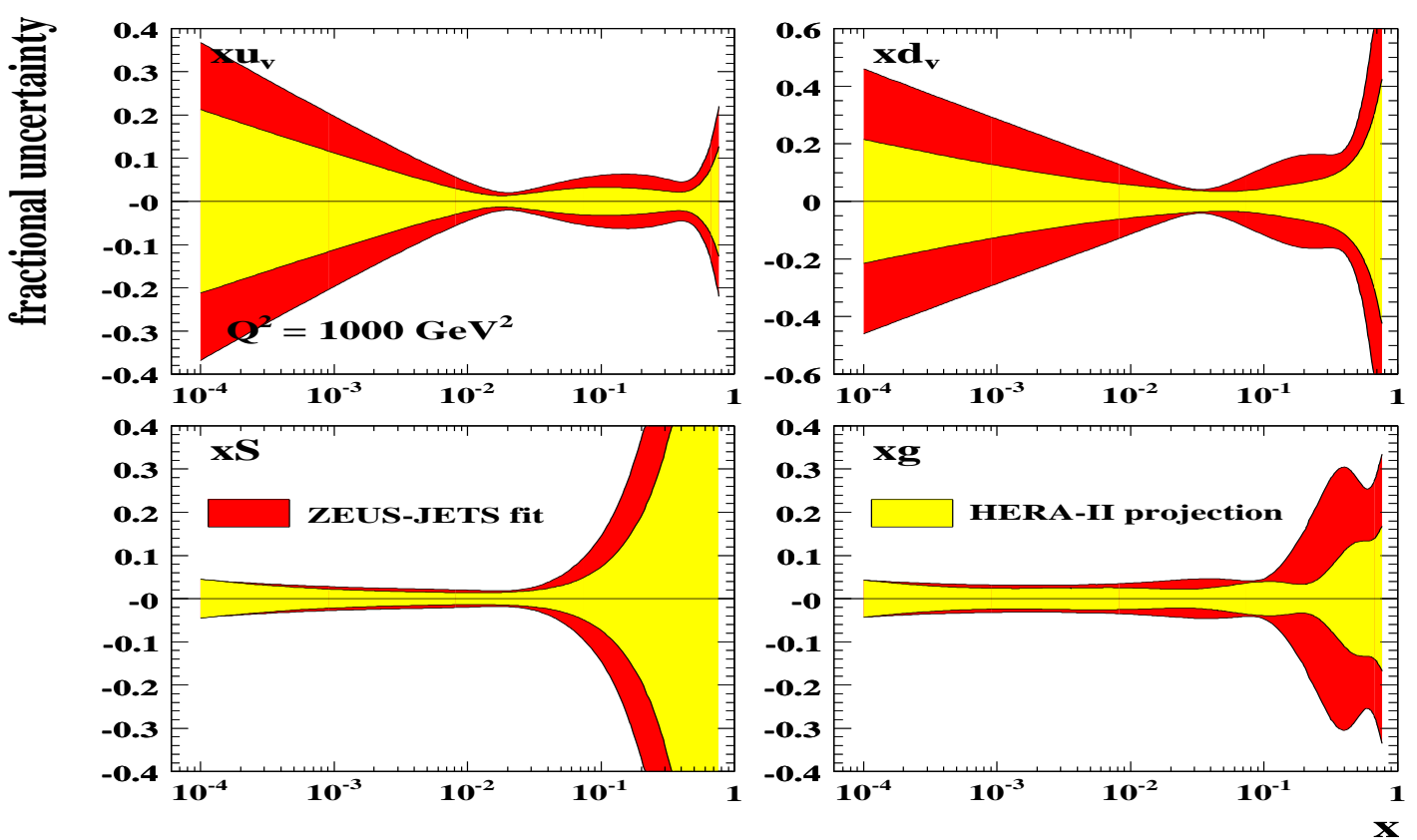

Fig. 2: The fractional PDF uncertainties, as a function of $x$, for the $u$-valence, $d$-valence, sea-quark and gluon distributions at $Q^{2}=1000 \mathrm{GeV}^{2}$. The red shaded bands show the results of the ZEUS-JETS fit and the yellow shaded bands show the results of the HERA-II projected fit.

from fits to HERA data alone, are largely limited by the statistical precision of existing measurements. Therefore, the impact of future data from HERA is likely to be most significant in fits to only HERA data.

\subsection{The ZEUS NLO QCD fit}

The ZEUS-JETS PDF fit has been used as the basis for all results shown in this paper. The most important details of the fit are summarised here. A full description may be found elsewhere [5]. The fit includes the full set of ZEUS inclusive neutral and charged current $e^{ \pm} p$ data from HERA-I (1994-2000), as well as two sets of high precision jet data in $e^{+} p$ DIS $\left(Q^{2}>>1 \mathrm{GeV}^{2}\right)$ and $\gamma p\left(Q^{2} \sim 0\right)$ scattering. The inclusive data used in the fit, span the kinematic range $6.3 \times 10^{-5}<x<0.65$ and $2.7<Q^{2}<30000$ $\mathrm{GeV}^{2}$.

The PDFs are obtained by solving the NLO DGLAP equations within the $\overline{\mathrm{MS}}$ scheme. These equations yield the PDFs at all values of $Q^{2}$ provided they are input as functions of $x$ at some starting scale $Q_{0}^{2}$. The resulting PDFs are convoluted with coefficient functions to give predictions for structure functions and, hence, cross sections. In the ZEUS fit, the $x u_{v}(x)$ (u-valence), $x d_{v}(x)$ ( $d$-valence), $x S(x)$ (total sea-quark), $x g(x)$ (gluon) and $x(\bar{d}(x)-\bar{u}(x))$ PDFs are parameterised at a starting scale of $Q_{0}^{2}=7$ $\mathrm{GeV}^{2}$ by the form,

$$
x f(x)=p_{1} x^{p_{2}}(1-x)^{p_{3}} P(x),
$$

where $P(x)=\left(1+p_{4} x\right)$. No advantage in the $\chi^{2}$ results from using more complex polynomial forms. The normalisation parameters, $p_{1}\left(u_{v}\right)$ and $p_{1}\left(d_{v}\right)$, are constrained by quark number sum rules while $p_{1}(g)$ is constrained by the momentum sum rule. Since there is no information to constrain any difference in the low- $x$ behaviour of the $u$ - and $d$-valence quarks, $p_{2}\left(u_{v}\right)$ has been set equal to $p_{2}\left(d_{v}\right)$. The data from HERA are currently less precise than the fixed target data in the high- $x$ regime. Therefore, the high$x$ sea and gluon distributions are not well constrained in current fits to HERA data alone. To account for this, the sea shape has been restricted by setting $p_{4}(S)=0$. The high- $x$ gluon shape is constrained 


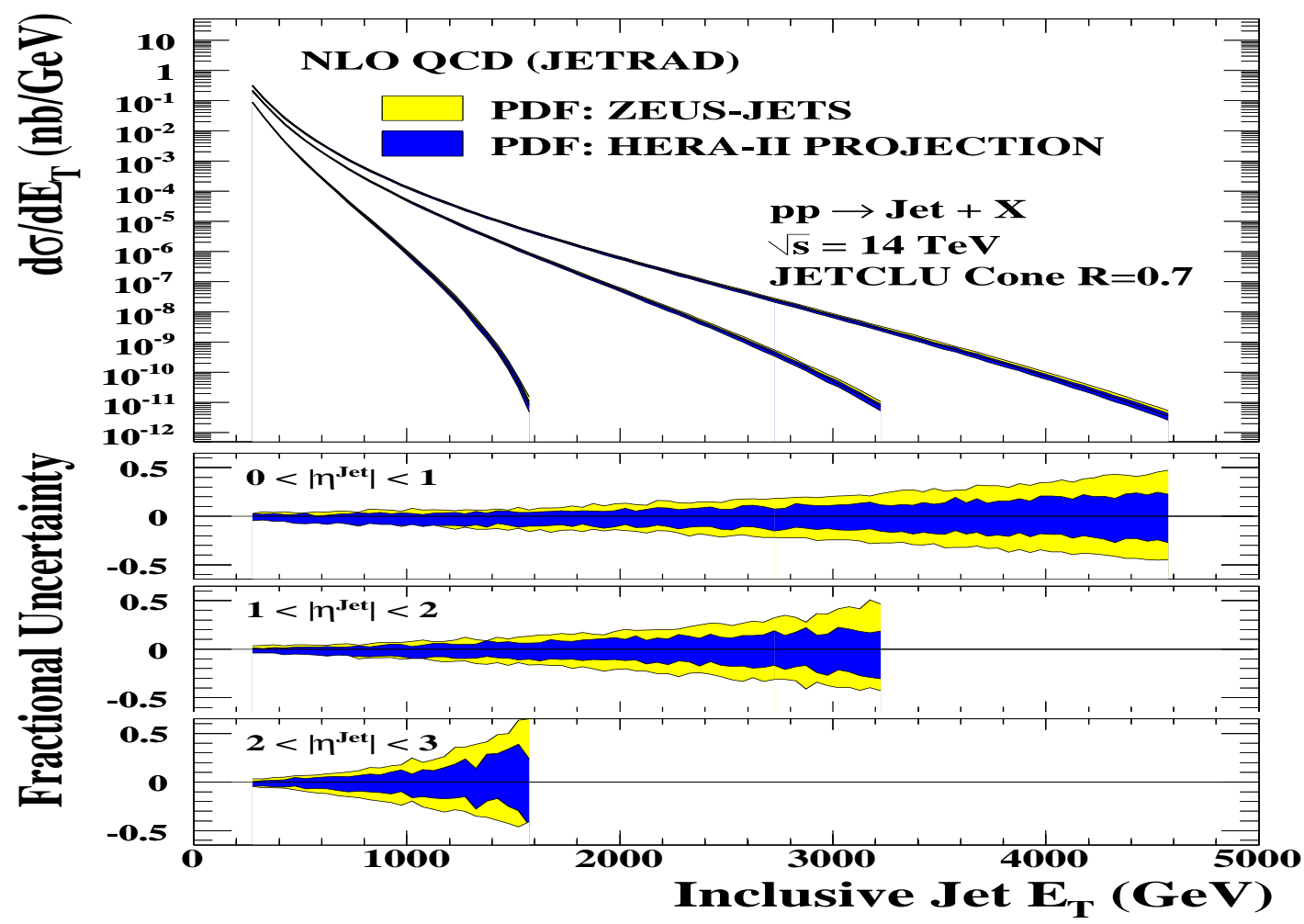

Fig. 3: NLO QCD inclusive jet cross section predictions at $\sqrt{s}=14 \mathrm{TeV}$ in three regions of pseudo-rapidity. The yellow and blue bands show the PDF uncertainties from the ZEUS-JETS and HERA-II projected fits, respectively.

by the inclusion of HERA jet data. In fits to only HERA data, there is no information on the shape of $\bar{d}-\bar{u}$. Therefore, this distribution has its shape fixed consistent with Drell-Yan data and its normalisation set consistent with the size of the Gottfried sum rule violation. A suppression of the strange sea with respect to the non-strange sea of a factor of 2 at $Q_{0}^{2}$ is also imposed, consistent with neutrino induced dimuon data from CCFR. The value of the strong coupling has been fixed to $\alpha_{s}\left(M_{\mathrm{Z}}\right)=0.1180$. After all constraints, the ZEUS-JETS fit has 11 free parameters. Heavy quarks were treated in the variable flavour number scheme of Thorne \& Roberts [15]. Full account was taken of correlated experimental systematic uncertainties, using the Offset Method [3,16].

\section{Results and discussion}

In this section, the results of two separate studies are presented. The first study provides an estimate of how well the PDF uncertainties may be known by the end of HERA-II, within the currently planned running scenario, while the second study investigates the impact of a future HERA measurement of $\mathrm{F}_{\mathrm{L}}$ on the gluon distribution. All results presented, are based on the recent ZEUS-JETS PDF analysis [5], as described in the previous section.

\subsection{PDF uncertainty estimates for the end of HERA running}

The data from HERA-I are already very precise and cover a wide kinematic region. However, HERAII is now running efficiently and is expected to provide a substantial increase in luminosity. Current estimates suggest that, by the end of HERA running (in mid-2007), an integrated luminosity of 700 $\mathrm{pb}^{-1}$ should be achievable. This will allow more precise measurements of cross sections that are curently statistically limited: in particular, the high- $Q^{2} \mathrm{NC}$ and $\mathrm{CC}$ data, as well as high- $Q^{2}$ and/or high- $E_{\mathrm{T}}$ jet data. In addition to the simple increase in luminosity, recent studies [17] have shown that future jet cross 
section measurements, in kinematic regions optimised for sensitivity to PDFs, should have a significant impact on the gluon uncertainties. In this paper, the effect on the PDF uncertainties, of both the higher precision expected from HERA-II and the possibility of optimised jet cross section measurements, has been estimated in a new QCD fit. This fit will be referred to as the "HERA-II projection".

In the HERA-II projected fit, the statistical uncertainties on the currently available HERA-I data have been reduced. For the high- $Q^{2}$ inclusive data, a total integrated luminosity of $700 \mathrm{pb}^{-1}$ was assumed, equally divided between $e^{+}$and $e^{-}$. For the jet data, an integrated luminosity of $500 \mathrm{pb}^{-1}$ was assumed. The central values and systematic uncertainties were taken from the published data in each case. In addition to the assumed increase in precision of the measurements, a set of optimised jet cross sections were also included, for forward dijets in $\gamma p$ collisions, as defined in a recent study [17]. Since no real data are yet available, simulated points were generated using the NLO QCD program of FrixioneRidolfi [18], using the CTEQ5M1 [19] proton and AFG [20] photon PDFs. The statistical uncertainties were taken to correspond to $500 \mathrm{pb}^{-1}$. For this study, systematic uncertainties on the optimised jet cross sections were ignored. The simulated optimised jet cross section points, compared to the predictions of NLO QCD using the ZEUS-S proton PDF [21], are shown in Fig. 11

Table 1 lists the data-sets included in the ZEUS-JETS and HERA-II projected fits. The luminosities of the (real) HERA-I measurements and those assumed for the HERA-II projection are also given.

The results are summarised in Fig. 2. which shows the fractional PDF uncertainties, for the $u$ - and $d$-valence, sea-quark and gluon distributions, at $Q^{2}=1000 \mathrm{GeV}^{2}$. The yellow bands show the results of the ZEUS-JETS fit while the red bands show those for the HERA-II projection. Note that the same general features are observed for all values of $Q^{2}$. In fits to only HERA data, the information on the valence quarks comes from the high- $Q^{2} \mathrm{NC}$ and $\mathrm{CC}$ cross sections. The increased statistical precision of the high- $Q^{2}$ data, as assumed in the HERA-II projected fit, gives a significant improvement in the valence uncertainties over the whole range of $x$. For the sea quarks, a significant improvement in the uncertainties at high- $x$ is also observed. In contrast, the low- $x$ uncertainties are not visibly reduced. This is due to the fact that the data constraining the low- $x$ region tends to be at lower- $Q^{2}$, which are already systematically limited. This is also the reason why the low- $x$ gluon uncertainties are not significantly reduced. However, the mid-to-high- $x$ gluon, which is constrained by the jet data, is much improved in the HERA-II projected fit. Note that about half of the observed reduction in the gluon uncertainties is due to the inclusion of the simulated optimised jet cross sections.

\subsubsection{Inclusive jet cross sections at the LHC}

The improvement to the high- $x$ partons, observed in the HERA-II projection compared to the ZEUSJETS fit, will be particularly relevant for high-scale physics at the LHC. This is illustrated in Fig. 3 which shows NLO QCD predictions from the JETRAD [22] programme for inclusive jet production at $\sqrt{s}=14 \mathrm{TeV}$. The results are shown for both the ZEUS-JETS and the HERA-II projected PDFs. The uncertainties on the cross sections, resulting from the PDFs, have been calculated using the LHAPDF interface [23]. For the ZEUS-JETS PDF, the uncertainty reaches $\sim 50 \%$ at central pseudo-rapidities, for the highest jet transverse energies shown. The prediction using the HERA-II projected PDF shows a marked improvement at high jet tranverse energy.

\subsection{Impact of a future HERA measurement of $F_{\mathrm{L}}$ on the gluon PDF}

The longitudinal structure function, $\mathrm{F}_{\mathrm{L}}$, is directly related to the gluon density in the proton. In principle, $\mathrm{F}_{\mathrm{L}}$ can be extracted by measuring the NC DIS cross section at fixed $x$ and $Q^{2}$, for different values of $y$ (see Eqn. 11. A precision measurement could be achieved by varying the centre-of-mass energy, since $s=Q^{2} / x y \approx 4 E_{e} E_{p}$, where $E_{e}$ and $E_{p}$ are the electron and proton beam energies, respectively. Studies [24] have shown that this would be most efficiently achieved by changing the proton beam energy. However, such a measurement has not yet been performed at HERA.

There are several reasons why a measurement of $\mathrm{F}_{\mathrm{L}}$ at low- $x$ could be important. The gluon 


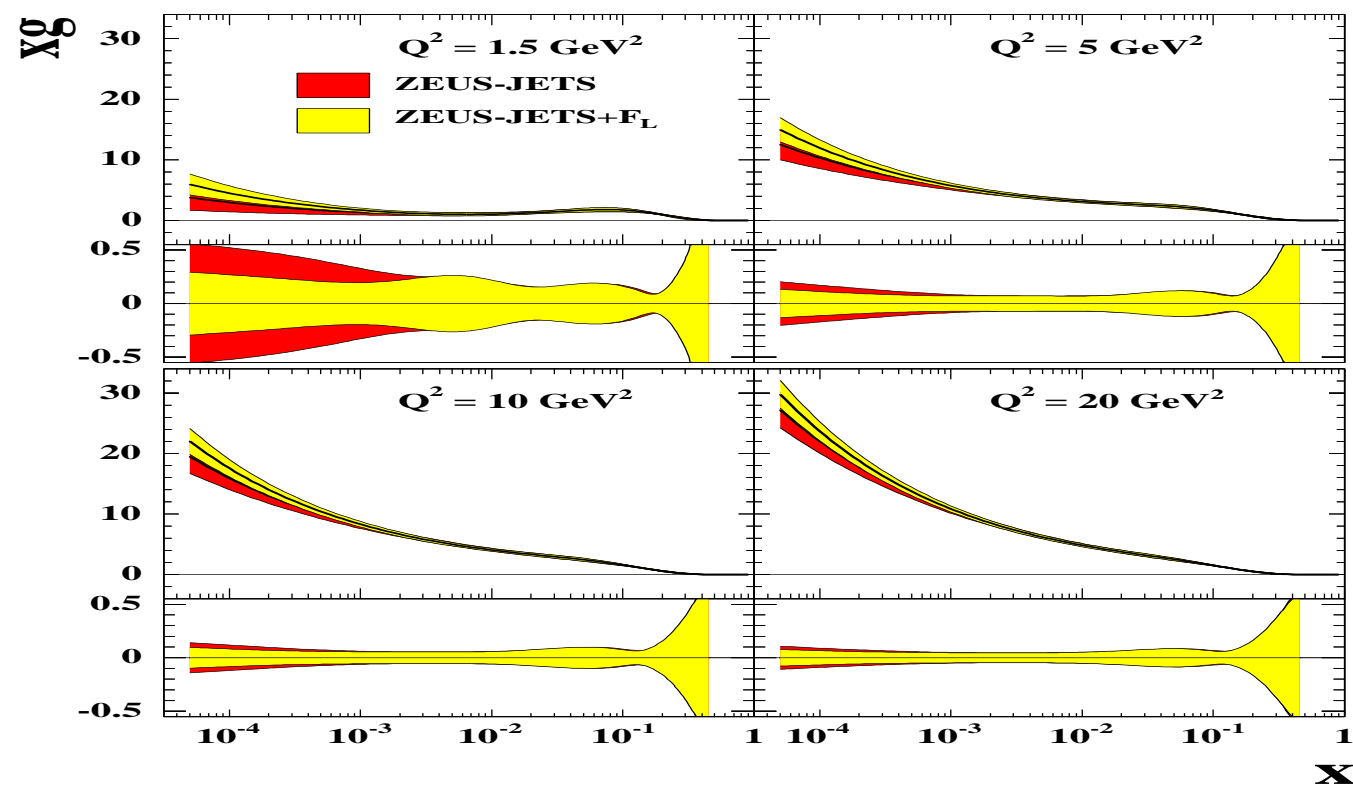

Fig. 4: The gluon PDFs, showing also the fractional uncertainty, for fits with and without inclusion of the simulated $\mathrm{F}_{\mathrm{L}}$ data, for $Q^{2}=1.5,5,10$ and $20 \mathrm{GeV}^{2}$. The red shaded bands show the results of the ZEUS-JETS fit and the yellow shaded band show the results of the ZEUS-JETS+F $\mathrm{L}$ fit.

density is not well known at low- $x$ and so different PDF parameterisations can give quite different predictions for $\mathrm{F}_{\mathrm{L}}$ at low- $x$. Therefore, a precise measurement of the longitudinal sturcture function could both pin down the gluon PDF and reduce its uncertainties. Furthermore, predictions of $\mathrm{F}_{\mathrm{L}}$ also depend upon the nature of the underlying theory (e.g. order in QCD, resummed calculation etc). Therefore, a measurement of $\mathrm{F}_{\mathrm{L}}$ could also help to discriminate between different theoretical models.

\subsubsection{Impact on the gluon PDF uncertainties}

The impact of a possible future HERA measurement of $\mathrm{F}_{\mathrm{L}}$ on the gluon PDF uncertainties has been investigated, using a set of simulated $\mathrm{F}_{\mathrm{L}}$ data-points [24]. The simulation was performed using the GRV94 [25] proton PDF for the central values, and assuming $E_{e}=27.6 \mathrm{GeV}$ and $E_{p}=920,575,465$ and $400 \mathrm{GeV}$, with luminosities of $10,5,3$ and $2 \mathrm{pb}^{-1}$, respectively. Assuming that the luminosity scales simply as $E_{p}^{2}$, this scenario would nominally cost $35 \mathrm{pb}^{-1}$ of luminosity under standard HERA conditions. However, this estimate takes no account of time taken for optimisation of the machine with each change in $E_{p}$, which could be considerable. The systematic uncertainties on the simulated datapoints were calculated assuming a $\sim 2 \%$ precision on the inclusive NC cross section measurement. A more comprehensive description of the simulated data is given elsewhere [24].

The simulated data were included in the ZEUS-JETS fit. Figure 4 shows the gluon distribution and fractional uncertainties for fits with and without inclusion of the simulated $\mathrm{F}_{\mathrm{L}}$ data. The results indicate that the gluon uncertainties are reduced at low- $x$, but the improvement is only significant at relatively low $Q^{2} \lesssim 20 \mathrm{GeV}^{2}$.

\subsubsection{Discrimination between theoretical models}

In order to assess whether a HERA measurement of $F_{L}$ could discriminate between theoretical models, two more sets of $F_{L}$ data-points have been simulated [26], using different theoretical assumptions. The first of the two sets was generated using the MRSG95 [27] proton PDF, which has a large gluon density. The PDFs were then convoluted with the NNLO order coefficient functions, which are large and positive. 


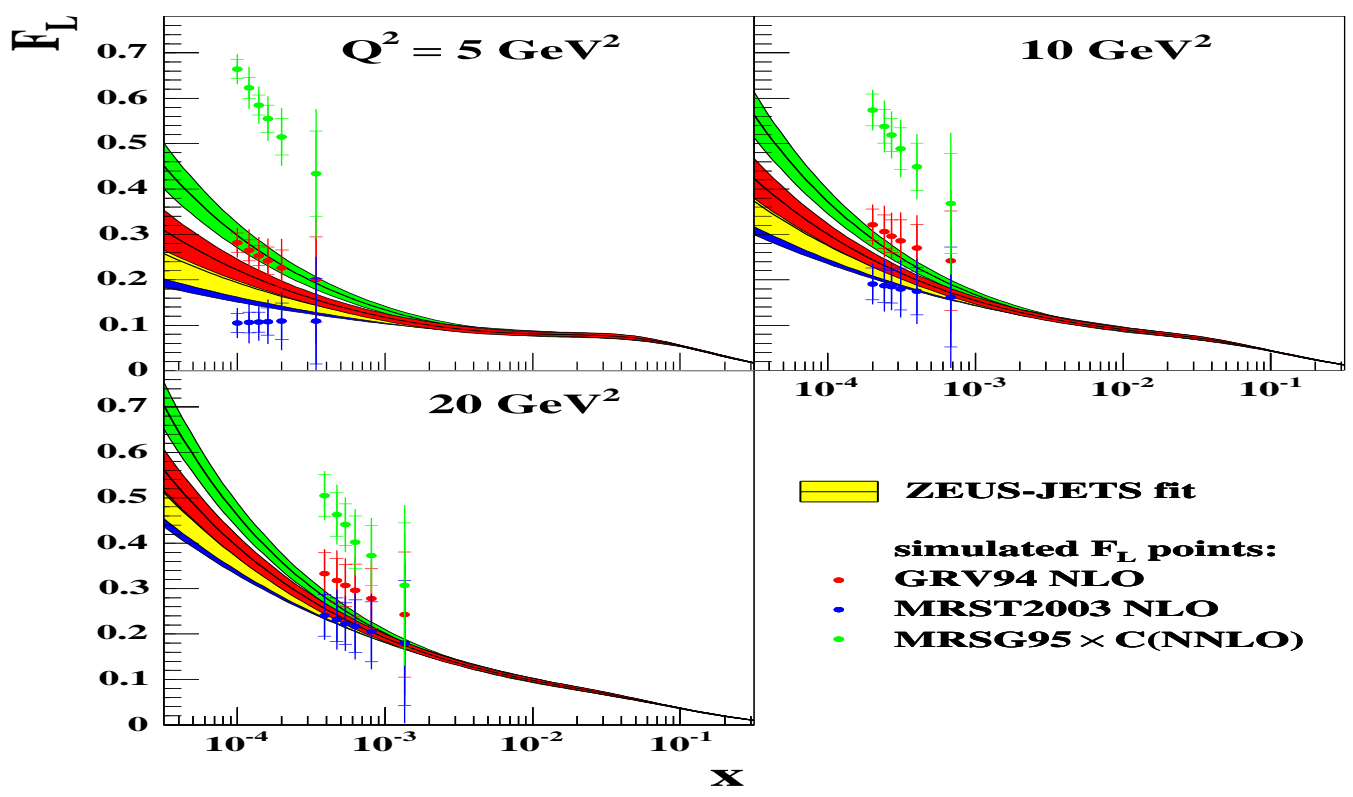

Fig. 5: The distribution of the longitudinal structure function $\mathrm{F}_{\mathrm{L}}$ at $Q^{2}=5,10$ and $20 \mathrm{GeV}^{2}$. The blue, red and green points show the simulated $\mathrm{F}_{\mathrm{L}}$ data-points, respectively labelled maximum, middle and minimum in Table 2 The blue, red and green shaded bands show the NLO QCD predictions, in the case where the data-points of the corresponding colour have been included in the fit. For comparison, the yellow shaded band shows the prediction of the ZEUS-JETS fit.

\begin{tabular}{lll}
\hline & PDF & QCD order of coefficient functions \\
\hline Maximum $F_{L}$ & MRSG95 & NNLO \\
Middle $F_{L}$ & GRV94 & NLO \\
Minimum $F_{L}$ & MRST2003 & NLO \\
\hline
\end{tabular}

Table 2: Summary of the PDFs used to generate the simulated $\mathrm{F}_{\mathrm{L}}$ data-points. The extreme maximum $F_{L}$ points were generated using the MRSG95 PDF, and convoluted with NNLO coefficient functions. The middle points were generated using the GRV94 PDF, and the extreme minimum points were generated using the MRST2003 PDF, which has a negative gluon at low- $x$.

This gives the "maximum" set of $\mathrm{F}_{\mathrm{L}}$ data-points. In contrast, the second set has been generated using the MRST2003 [28] proton PDF, which has a negative gluon at low- $x$ and low- $Q^{2}$, thus providing a "minimum" set of $F_{L}$ data. The original set of $F_{L}$ points described in the previous subsection lies between these two extremes. The details of all three sets are summarised in Tab. 2

Figure 5 shows the results of including, individually, each set of simulated $\mathrm{F}_{\mathrm{L}}$ data into the ZEUS NLO QCD fit. The results show that the NLO fit is relatively stable to the inclusion of the extreme sets of data. This indicates that a measurement of $\mathrm{F}_{\mathrm{L}}$ could discriminate between certain theoretical models. However, it should be noted that the maximum and minimum models studied here were chosen specifically to give the widest possible variation in $\mathrm{F}_{\mathrm{L}}$. There are many other alternatives that would lie between these extremes and the ability of an $\mathrm{F}_{\mathrm{L}}$ measurement to discriminate between them would depend both on the experimental precision of the measurement itself, as well as the theoretical uncertainties on the models being tested.

\section{Acknowlegdements}

The authors wish to thank M. Klein and $\mathrm{R}$. Thorne for providing the $\mathrm{F}_{\mathrm{L}}$ predictions, as well as for useful discussions. C. Gwenlan wishes to thank PPARC for the support of this work. 


\section{References}

[1] A. D. Martin et al., Eur. Phys. J. C23, 73 (2002).

[2] J. Pumplin et al., JHEP 0207, 012 (2002).

[3] ZEUS Coll., S. Chekanov et al., Phys. Rev. D67, 012007 (2003).

[4] G. Altarelli, G. Parisi, Nucl. Phys. B126, 298 (1977);

V. N. Gribov, L. N. Lipatov, Sov. J. Nucl. Phys. 15438 (1972);

L. N. Lipatov, Sov. J. Nucl. Phys. 20, 94 (1975);

Yu. L. Dokshitzer, JETP 46, 641 (1977).

[5] ZEUS Coll., S. Chekanov et al., Eur. Phys. J. C42, 1 (2005).

[6] H1 Coll., C. Adloff et al., Eur. Phys. J. C30, 32 (2003).

[7] ZEUS Coll., S. Chekanov et al., Eur. Phys. J. C 21, 443 (2001).

[8] ZEUS Coll., S. Chekanov et al., Eur. Phys. J. C 28, 175 (2003).

[9] ZEUS Coll., S. Chekanov et al., Preprint hep-ex/0401003, (2004).

[10] ZEUS Coll., J. Breitwig et al., Eur. Phys. J. C 12, 411 (2000).

[11] ZEUS Coll., S. Chekanov et al., Phys. Lett. B 539, 197 (2002).

[12] ZEUS Coll., S. Chekanov et al., Eur. Phys. J. C 32, 16 (2003).

[13] ZEUS Coll., S. Chekanov et al., Phys. Lett. B 547, 164 (2002).

[14] ZEUS Coll., S. Chekanov et al., Eur. Phys. J. C 23, 615 (2002).

[15] R. S. Thorne and R. G. Roberts, Phys. Rev. D 57, 6871 (1998).

[16] A. M. Cooper-Sarkar, J. Phys. G28, 2669 (2002).

[17] C. Targett-Adams, private communication.

[18] S. Frixione and G. Ridolfi, Nucl. Phys., B507, 315 (1997);

S. Frixione, Nucl. Phys., B507, 295 (1997).

[19] CTEQ Coll., H. L. Lai et al., Eur. Phys. J. C12, 375 (2000);

CTEQ Coll., H. L. Lai et al., Phys. Rev. D51, 4763 (1995).

[20] P. Aurenche, J. Guillet, M. Fontannaz, Z. Phys. C64, 621 (1994).

[21] ZEUS Coll., Phys. Rev. D67 (2003) 012007.

[22] W. T. Giele, E. W. N. Glover, David A. Kosower, Nucl. Phys.B403, 633 (1993).

[23] M. R. Whalley, D. Bourilkov, R. C. Group, Les Houches Accord Parton Density Function Interface, hep-ph/0508110 [http://durpdg.dur.ac.uk/lhapdf; http://hepforge.cedar.ac.uk/lhapdf/)].

[24] M. Klein, "Future $F_{\mathrm{L}}$ at HERA", in proceedings, DIS04 (2004).

[25] M.Gluck, E. Reya and A. Vogt, Z. Phys. C67, 433 (1995).

[26] R. Thorne, private communication.

[27] A. D. Martin, W. J. Stirling and R. G. Roberts, Phys. Lett., B354, 155 (1995).

[28] A. D. Martin, R. G. Roberts, W. J. Stirling and R. S. Thorne, Eur. Phys. J., C35, 325 (2004). 